\title{
Téoros
}

Revue de recherche en tourisme

\section{La fin des ruines}

\section{Martin Drouin}

Volume 25, numéro 1, printemps 2006

URI : https://id.erudit.org/iderudit/1071037ar

DOI : https://doi.org/10.7202/1071037ar

Aller au sommaire du numéro

\section{Éditeur(s)}

Université du Québec à Montréal

\section{ISSN}

0712-8657 (imprimé)

1923-2705 (numérique)

Découvrir la revue

\section{Citer cette note}

Drouin, M. (2006). La fin des ruines. Téoros, 25(1), 58-61.

https://doi.org/10.7202/1071037ar d'utilisation que vous pouvez consulter en ligne.

https://apropos.erudit.org/fr/usagers/politique-dutilisation/ 


\section{La fin des ruines}

\section{Martin Drouin}

Qui n'a pas imaginé, enfant, la silhouette sombre d'une maison abandonnée? Derrière un grillage de fer fatigué se dressait l'étrange figure, éclairée par une lumière blafarde qui laissait deviner des vitres brisées et des murs abîmés. Cette vision s'accompagnait généralement du sifflement du vent ponctué par le battement sordide des volets. Une maison jadis habitée par des propriétaires à demi-oubliés et que la légende avait métamorphosés en seigneurs excentriques. Qui n'a pas rêvé d'y entrer, aspiré par une aura mystérieuse ? L'image de ce lieu qui habita mon enfance et que je cherchais inconsciemment dès lors que je découvrais de nouveaux quartiers a été reprise par de nombreux films, qui l'ont presque transformée en cliché... à moins que ce ne soit l'inverse. Quoi qu'il en soit, son emploi exprime à merveille la force des édifices abandonnés dans l'imaginaire occidental. Une question m'accompagne encore aujourd'hui : pourrais-je un jour visiter ce lieu?

Cette vision possède une contrepartie aux allures plus nobles : la ruine de pierres. II me fallut être plus âgé pour laisser mon esprit vagabonder sur les restes de ces bâtiments, témoins d'anciennes civilisations, et ainsi délaisser, pour un moment, une architecture familière. Des noms prestigieux se bousculaient et me transportaient alors vers des mondes lointains : la ville d'Angkor envahie d'une végétation luxuriante, l'ancienne Babylone des rois perses Hammourabi et Nabuchodonosor, les temples et les nécropoles de Louxor, la cité inca de Machu Picchu, la roche sculptée de Pétra et les restes de la mythique Troie. Leurs seuls rappels permettent encore de raviver les images des livres parcourus à défaut de suivre réellement les pas des aventuriers des siècles passés. La peinture et la littérature ont, semblablement au cinéma, permis à ces lieux de connaître une fortune critique. Je les visiterai peut-être un jour, désormais inscrits sur les itinéraires touristiques et les hauts lieux du patrimoine mondial. Je ne me doutais cependant pas que ces figures que je viens d'évoquer participaient à un même phénomène : le pouvoir d'évocation d'un ailleurs historique.

J'appris plus tard que le goût des ruines n'était pas universellement partagé. II était le fait de la civilisation dite occidentale et de son inclinaison pour l'observation de sa propre historicité. La ruine permettait en effet de mesurer, ou plutôt de confronter, scientifiquement ou empiriquement, sa position sur la ligne du temps par l'expérience du passé. De la même manière dont l'intérêt se circonscrivait géo-

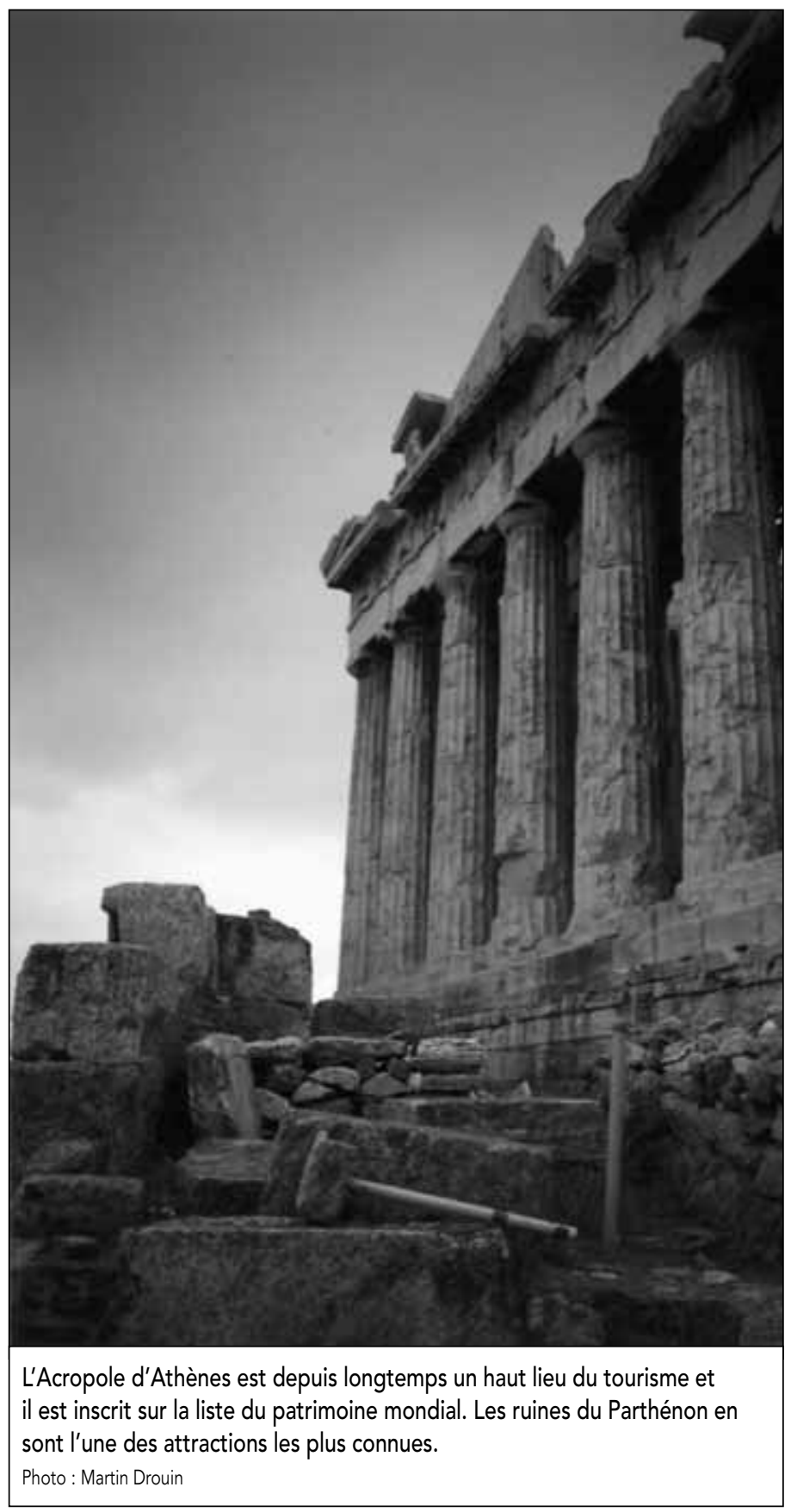


graphiquement, il était possible d'en dater l'apparition. La redécouverte de l'Antiquité par les humanistes de la Renaissance constituait la source généalogique de l'engouement ${ }^{1}$. Le besoin de se tourner vers les textes des grands auteurs s'accompagna d'un désir de côtoyer les lieux et les objets de la même époque. Les vestiges architecturaux devenaient alors le dépôt de l'histoire. Ils en étaient une trace, c'est-à-dire le «signe matériel d'une grandeur disparue et d'une perfection perdue » (Mortier, 1974 : 223) ${ }^{2}$. Je fréquentais déjà l'université lorsque je découvris que le monument historique - et par extension la notion de patrimoine - était apparu timidement à la même période, même si, comme l'a démontré Françoise Choay, l'intérêt ne se doublait pas, comme aujourd'hui, d'une nécessaire volonté de conservation des œuvres repérées (Choay, 1992) ${ }^{3}$. De l'ouvrage chargé d'histoire et de la curiosité pour la ruine découlait, pour certains, le rêve de les visiter; ce que réalisa à Rome, par exemple, Rabelais dans la décennie 1530 (Plattard, 1928 : 119-129). Ruine, patrimoine et appel du voyage s'unissaient alors pour une première fois. Le Forum, le Colisée, les Termes de Caracalla et bien d'autres vestiges allaient ainsi connaître en quelque sorte une seconde vie en voyant défiler des générations et des générations de voyageurs ; rituel que j'accomplis à mon tour lors de mon premier grand voyage à l'orée de ma vie d'adulte.

Au cours des premiers siècles, l'intérêt avait été essentiellement romain, puisque les humanistes y voyaient un idéal de perfection. Le développement du genre au XVIII siècle allait élargir le corpus. La Grèce, bien sûr, puis les vestiges des autres continents, retinrent l'attention. Dans leur sillage, les ruines rattachées à une histoire locale s'imposaient à mesure que se construisaient les identités nationales. Les découvertes du XIXe siècle allaient, à leur tour, multiplier les lieux d'observation. La colonne et le fronton dépecés retrouvant forme par l'anastylose, la ville biblique émergeant de la quiétude par fouilles successives et le donjon abandonné se côtoyaient au cœur d'une même passion. Deux attitudes surgissaient de la contemplation. L'une, archéologique, cherchait, à travers les fragments dévoilés, l'empreinte laissée par des sociétés disparues et à en reconstruire la grandeur. L'autre, esthétique, laissait le regard vagabonder sur les formes décharnées pour en expérimenter la beauté et le pittoresque. Toutes deux permettaient - et permettent encore - à l'observateur de communiquer avec l'histoire. Je goûtais beaucoup plus tard le double sentiment en séjournant plus d'une semaine à Hampi, dans le nord de l'État du Karnataka en Inde. L'ancienne capitale du royaume de Vijaynâgara, avec ses sept murailles concentriques et son demi-million d'habitants, était devenue un village paisible d'à peine 1000 habitants. La population indienne fréquentait encore le temple Virupaksha, dédié à Shiva, tandis que les touristes découvraient, chacun à son rythme, les vingt-six kilomètres carrés de ruines ${ }^{4}$. Avec ma carte et mes livres, je tentais de repérer et de reconstruire la splendeur passée et d'imaginer la vie oubliée dans ce paysage aujourd'hui désertique composé de gigantesques rochers arrondis d'où émergeaient, ici et là, les restes du bain de la reine ou les étables des éléphants qui renvoyaient à l'une des plus fastueuses périodes de l'Inde.

Dans cette histoire de la ruine, l'intérêt connut son apogée au XIX siècle. L'invention du monument historique et les réflexions qui l'accompagnèrent bousculèrent cependant leur avenir. Dans ce bouillonnement d'idées, deux positions antinomiques départagèrent les attitudes quant aux traitements des témoins architecturaux de l'histoire.

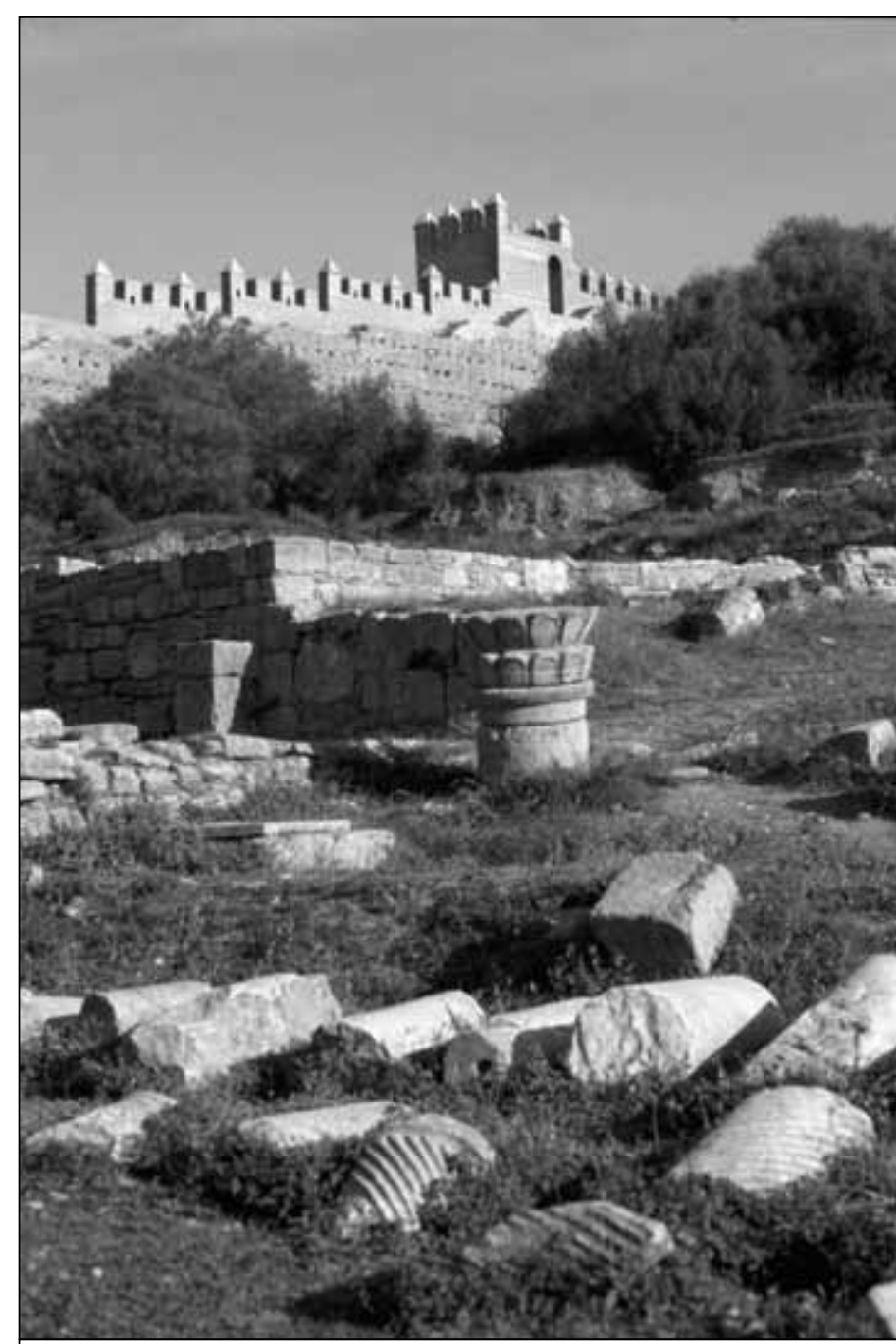

Les restes de l'ancienne ville romaine de Sala Colonia sont enserrés par les murs de la nécropole de Chella près de Rabat. Certains guides touristiques présentent le site comme l'un des plus romantiques du Maroc. Photo : Martin Drouin

Elles s'incarnent aujourd'hui par deux personnages : John Ruskin ${ }^{5}$ et Viollet-le-Duc ${ }^{6}$. Le premier soutenait un non-interventionnisme qui célébrait le passage du temps : "the greatest glory of a building is not its stones, nor its gold. Its glory is in its Age [...] "7 . La vénérable valeur d'âge, que l'historien autrichien Aloïs Riegl (1903) allait définir quelques années plus tard, trouvait chez Ruskin sa quintessence. La corrosion du temps, celle qui donnait à l'objet toute sa patine, permettait de véritablement mesurer et de goûter la valeur du monument. La deuxième personnalité pourrait à l'inverse être qualifiée d'interventionniste. La restauration devait redonner au monument la figure et les caractéristiques qui l'avaient vu naître. Ainsi, tel qu'il est souvent présenté selon Viollet-le-Duc, "Restaurer un édifice, c'est le rétablir dans un état complet qui peut n'avoir jamais existé à un moment donné " 8 . L'un des exemples les plus évocateurs est probablement le château de Pierrefonds. La forteresse médiévale, qui avait été détruite sur l'ordre de Louis XIII au cours des guerres de religion et laissée en ruines pendant deux cents ans, fut restaurée par Viollet-le-Duc à la demande de Napoléon III, qui s'enticha du lieu et 
souhaita le transformer en une résidence impériale. Après avoir traversé la forêt de Compiègne, le touriste se retrouve dans le petit bourg qui encercle le château et saisit alors toute la magnificence de l'œuvre de l'architecte restaurateur, dont les travaux s'étalèrent de 1853 à 1885. Murs d'enceinte majestueux, sculptures monumentales, bas-reliefs ornés d'entrelacs et illustrés de bestiaires : bref, tous les talents furent mis à contribution pour recréer l'image du (ou d'un) château ${ }^{9}$. À l'inverse, pour poursuivre avec le cas français, les dépouilles des forteresses cathares illustrent le noninterventionnisme - bien sûr contrôlé - à la Ruskin. Si le circuit peut débuter à Carcassonne, ville médiévale fortifiée et lourdement restaurée par le même Viollet-le-Duc, les châteaux suivants de Mirepoix, de Montségur, de Peyrepertuse, de Quéribus, de Roquefixade, pour ne nommer que ceux-là, n'offrent aux visiteurs que des ruines. Pourtant, dans un décor époustouflant, la silhouette d'une ancienne tour, de murs aux trois quarts disparus, les restes d'une cour intérieure bercent et transportent le voyageur dans une histoire à demi légendaire que guide son imagination.

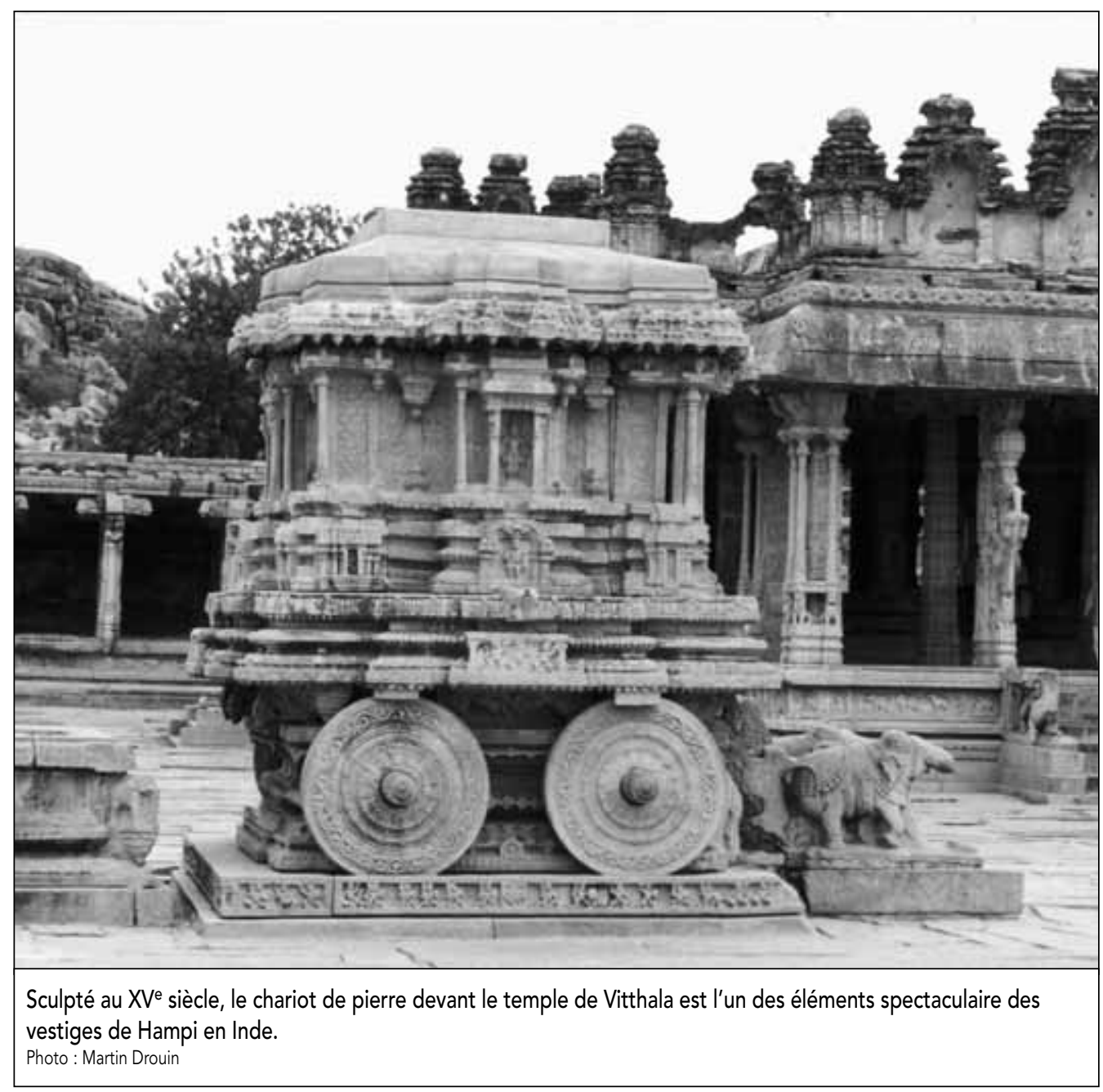

La démocratisation du tourisme au $X X^{e}$ siècle permit de multiplier les visiteurs qui eurent le bonheur de partager la magie de la ruine. Cependant, force est de constater que, parallèlement à l'extension des publics, le corpus des lieux à visiter s'est désormais stabilisé. Peu de ruines s'ajoutent aujourd'hui à la liste. Serait-ce la fin de la ruine ? Depuis un demi-millénaire le voyageur occidental s'était graduellement offert de nouvelles trouvailles; et qu'en est-il aujourd'hui ? La ruine n'est généralement plus qu'un état temporaire avant la restauration ou la démolition. Ainsi, pour reprendre Marc Augé, nous vivrions dans un monde où « les décombres n'ont plus le temps de devenir des ruines » $(2003: 9)^{10}$. Paradoxalement, des ruines, le siècle dernier en a abondamment produites. Les destructions des deux guerres mondiales en fournissent déjà un parfait exemple. Ce ne fut généralement qu'un état transitoire puisque les décennies suivantes virent les reconstructions se multiplier. Si les quelques piliers et les pans de murs de la cathédrale de Coventry ont été conservés en guise de témoignage, les Allemands au contraire célébraient dignement, l'année dernière à Dresde, l'inauguration de la Frauenkirche, monument baroque du XVIII ${ }^{\mathrm{e}}$ siècle reconstruit à l'identique après cinquante ans d'attente. Le spectacle des ruines ne serait-il pas un constant rappel de l'horreur de la guerre et des conséquences des bombardements dont le souvenir serait trop cruel ? Chaque collectivité tente d'y répondre à sa façon.
Dès lors que les bâtiments appartenaient à une époque plus récente, ma quête des ruines se voyait difficilement satisfaite. Pourtant, nombre de vestiges laissaient encore place à une certaine fréquentation. Parmi eux, les ruines de la société industrielle et les villages fantômes attiraient plus particulièrement mon attention. Entre la désaffection et la requalification, les friches étaient laissées à la merci de la population urbaine qui, par de multiples interventions, se les réappropriait. Les artistes, par exemple, en utilisent la poétique pour présenter des œuvres ou des événements, comme à Montréal, où le silo n` 5 se transforma en instrument de musique grandeur nature - le silophone - que les visiteurs avaient la charge de faire jouer ${ }^{11}$; ou à Douarnenez, lorsque l'ancienne conserverie de sardines Quéré, sur le point d'être reconvertie en médiathèque, prêta ses murs éventrés à l'exposition du peintre Paul Bloas. Dans les deux cas, le caractère éphémère et marginal de l'intervention renforçait l'état transitoire et exceptionnel de la ruine. En effet, seul le contre-emploi artistique des bâtiments abandonnés en autorisait une visite officielle.

Les vestiges du second type appelaient une tout autre approche, voire une réflexion nouvelle. "Tombeaux de bois ", les villages fantômes ne pouvaient s'offrir le luxe d'un abandon total ${ }^{12}$. La tentation de leur rendre vie n'était alors pas si loin. Au Québec, le village de Val-Jalbert faisait l'objet d'un plan de restauration progressif qui visait à maintenir les bâtiments en état, tout en laissant à la vue du visiteur la dé- 
gradation réelle des lieux. À l'opposé, l'ancien village minier de Virginia city, au Nevada, faisait revivre en son sein, à la manière d'un théâtre, les scènes du temps passé. Si la visite pouvait s'avérer instructive, elle occultait partiellement ce qui faisait le cœur même de la structure touristique. Seule l'animation justifiait la rencontre du visiteur avec la ruine.

Y aurait-il une incompatibilité entre la valeur heuristique de la ruine et la mise en exposition? Une peur que les supputations interprétatives du visiteur n'aient de limites que celles de sa propre culture (ou inculture) ? Si le message de la ruine n'était pas compris ou tout simplement amoindri? L'anachronisme pourrait alors se glisser sournoisement dans la lecture du visiteur. La ruine donne en effet à l'imagination un tremplin pour vagabonder, puisqu'elle procède davantage sur le mode de la suggestion et de la déambulation. Elle est une œuvre ouverte, pour reprendre la terminologie d'Umberto Eco (1979). Or, la mise en exposition n'est-elle pas justement la proposition d'un récit singulier qui offrirait au visiteur la lecture d'un concepteur? Bien que la médiation d'un message précis à travers des parcours réfléchis soit d'un grand support pour la compréhension du vestige, elle bride le visiteur dans sa capacité d'interprétation. La ruine se transforme alors en une œuvre fermée qui accepte difficilement les rêveries et les digressions du promeneur. Dans ce contexte, l'imagination n'est-elle pas comprise comme une source d'extrapolation et de fabulation?

"Contempler les ruines, ce n'est pas faire un voyage dans l'histoire, mais faire l'expérience du temps, du temps pur », écrivait Marc Augé (2003 : 38). C'est ce que, consciemment ou inconsciemment, le développement du genre depuis le $X V^{e}$ siècle a permis de consacrer. La ruine témoigne, à travers le fragment, d'une intégrité disparue, de l'impuissance des hommes, de l'usure, de la dévastation et de l'irréversibilité de l'histoire. Notre société actuelle serait-elle capable d'un tel regard sur elle-même? La vague patrimoniale actuelle avec son concert de requalification, de restauration et de rénovation, bien que nourrie de nobles intentions dans sa volonté de conservation, n'a pas encore accepté l'imperfection de la ruine dans son paysage. Je ne retrouverai probablement jamais la silhouette de la maison abandonnée de mon enfance. Aura-t-elle été démolie pour laisser place à un nouvel édifice? Aura-t-elle retrouvé une nouvelle jeunesse grâce au dévouement de propriétaires attentionnés ? Seul l'avenir laissera le temps d'y répondre.

Martin Drouin est historien. I/ est coordonnateur de l'Institut du patrimoine de l'UQAM.

\section{Notes}

1 Pour plus de détails, on peut consulter avec plaisir l'ouvrage de Sabine Foreno-Mendoza (2002).

2 Une approche structuraliste peut aujourd'hui rebuter le lecteur, mais l'ouvrage demeure une formidable source d'information.

3 L'historienne consacre deux longs chapitres à cette question.

4 Le gouvernement indien décidait en 2002 de faire de Hampi une destination internationale (portail officiel de l'État du Karnataka).

5 John Ruskin (1819-1900) publia de nombreux ouvrages, dont son plus célèbre, intitulé Les sept lampes de l'architecture (Ruskin, 1880).

6 Eugène Emmanuel Viollet-le-Duc (1814-1879) travailla à de nombreuses restaurations et publia un nombre considérable de textes (consulter à ce propos, Leniaud, 1994).
7 L'auteur poursuivait: "[...] and in that deep sense of voicefulness, of stern watching, of mysterious sympathy, nay, even of approval or condemnation, which we feel in walls that have long been washed by the passing waves of humanity» (Ruskin, 1880 : 187 ; Wheeler, 1992: 77-97).

8 Cette position, tout comme celle de Ruskin, devraient être évidemment nuancées (Viollet-le-Duc, Eugène-Emmanuel, 1854), Dictionnaire raisonné de l'architecture française $d u X I^{e}$ au XVIe siècle, Paris, Édition A. Morel, 1875-1889, p. 14, cité par Choay, 1992 : 116).

9 Classé monument historique, le château de Pierrefonds est régi par le Centre des monuments nationaux, qui l'ouvre aux visiteurs (site Internet de Monum).

10 L'anthropologue français offre une réflexion touffue et stimulante.

11 Un observatoire sonore a ainsi été aménagé dans le Vieux-Port de Montréal (site Internet de l'association Silophone).

12 Les maisons de l'aventure coloniale nord-américaine, tout comme les restes d'une abbaye du XIII ${ }^{\mathrm{e}}$ siècle, nécessitent un entretien constant. On peut consulter Coomans (2005: 47-57).

\section{Bibliographie}

Augé, Marc (2003), Le temps en ruines, Paris, Galilée.

Choay, Françoise ([1992] nouv. éd. 1999), L'allégorie du patrimoine, Paris, Seuil, p. 25-72.

Coomans, Thomas (2005), «From Romanticism to New Age: The Evolving Perception of a Church Ruin ", Téoros, vol. 24, n² 2, p. 47-57.

Eco, Umberto (1965), L'œuvre ouverte, Paris, Seuil, 1979, 315 p.

Foreno-Mendoza, Sabine (2002), Le temps des ruines. Le goût des ruines et les formes de la conscience historique à la Renaissance, Seysell, Éditions Champ Vallon, $221 \mathrm{p}$.

Jackson, Guy (2005), « Dresde fête la reconstruction de son joyau baroque », Le Devoir, 31 octobre, p. B8.

Karnataka, État du, [http://www.karnataka.com/tourism/hampi/] (consulté le 15 février 2006).

Leniaud, Jean-Michel (1994), Viollet-le-Duc ou les délires du système, Paris, Mengès, $225 \mathrm{p}$.

Monum, Centre des monuments nationaux, [www.monum.fr] (consulté le 15 février 2006).

Mortier, Roland (1974), La poétique des ruines en France, Genève, Librairie Droz.

Plattard, Jean (1928), Vie de François Rabelais, Paris / Bruxelles, Éditions G. Van Oest.

Riegl, Aloïs ([1903] trad. et publ. 1984), Le culte moderne des monuments. Son essence et sa genèse, Paris, Seuil, $125 \mathrm{p}$.

Ruskin, John ([1880] 1989), The Seven Lamps of Architecture, New York, Dover Publications, republication, $222 \mathrm{p}$.

Silophone, [www.silophone.net] (consulté le 15 février 2006).

Wheeler, Michael (1992), "Ruskin Among the Ruins: Tradition and the Temple ", dans Michael Wheeler et Nigel Whiteley (dir.), The Lamp of Memory. Ruskin, Tradition and Architecture, Manchester / New York, Manchester University Press, p. 77-97. 